\title{
Origins of Bipedalism
}

\author{
Kwang Hyun Ko* \\ Hanyang University, South Korea
}

\begin{abstract}
This article aimed to review various theories of bipedalism and provide a holistic answer to human evolution. There have been two questions regarding bipedalism: i) why were the earliest hominins partially bipedal?, and ii) why did hominins become increasingly bipedal over the time and replaced their less bipedal ancestors? To answer these questions, the prominent theories in the field, such as the savanna-based theory, the postural feeding hypotheses, and the provisioning model, are collectively examined. Because biological evolution is not a simple causation; there may be multiple answers to the evolution of bipedalism. The postural feeding hypothesis (reaching for food/balancing) provides an explanation for the partial bipedalism of the earliest hominins. The savannah-based theory describes how the largely bipedal hominins that started to settle on the ground became increasingly bipedal. The provisioning model (food-gathering/monogamy) explains questions arising after the postural feeding hypothesis and before the savannah theory in an evolutionary timeline. Indeed, there are no straight lines between the theories, and multiple forces could have pushed the evolution of bipedalism at different points. Finally, the arboreal hominins that possessed ambiguous traits of bipedalism were eliminated through the choice and selection. Using the biological analogy of the okapi and giraffe, efforts were put to explain how one of the branches (Homo) became increasingly bipedal, while the other (Pan) adapted to locomotion for forest life by narrowing the anatomical/biological focus in evolution.
\end{abstract}

Key words: locomotion, provisioning model, bipedalism, chimps, savannah; Origins of Bipedalism

\section{INTRODUCTION}

Bipedalism is an essential adaptation of the Hominin progeny that is considered the major force behind several skeletal changes shared by all the bipedal hominins (Lovejoy 1988). There are different hypotheses that explain how and why bipedalism evolved in humans. Similarly, the timing of the evolution of bipedalism is debated. The possible reasons for the evolution of human bipedalism include the freeing of the hands to use and carry tools, threat displays, sexual dimorphism in food gathering, and changes in climate and habitat (from jungle to savanna).

\section{Bipedalism: New Perspective}

As in other species, several characteristics of the ape-like hominin ancestors were advantageous for their survival. Human bipedalism was driven by the simple Darwinian principle of natural selection. Hominins did not consciously become bipedal for a specific reason. Instead, eons of time allowed the evolution of bipedalism in humans because it was a favorable trait (Auletta et al. 2011). Specifically, a distinctive set of observable traits in each species constitute characteristics that have lasted through natural selection out of the countless mutation traits that were observed during the timeline of the species (Ayala 2007).

This issue becomes more complex to investigate the actual process of 'Natural Selection', or

*Author for correspondence: highway2@hanyang.ac.kr 
'Darwinism' because several factors intervene over millions of years (Darwin 1963). Such factors may be interaction with the animals, the avoidance of competition, and/or the effective protection of offspring. Natural selection may also be influenced by the changes in the environmental settings (Miller 1995). 'Natural selection' is, however, a vague term that includes several factors. But, because of the wide definition of Darwinism, it does not have to be a single discrete principle, or hypothesis that provides holistic explanations of how 'survival' occurs. Scholars tend to fixate on causation, where A caused B (in this case, B is bipedalism), and are currently attempting to find an exact answer for the cause of bipedalism. However, the principles of evolution do not necessarily operate like cause and effect. Biological evolution is an example of trial and error (Wright 1932), i.e., if a trait works, it remains. Again, various traits were observed during the evolutionary timeline, but only a few remained.

The reason why bipedalism remained was because it was beneficial for the efficient survival of both the unit itself and its offspring. There are several theories debating human bipedalism (Tuttle 2015). However, if the evidence explains how bipedalism helped human ancestors survive, it was sensible to believe that there could be multiple answers to the question of the evolution of bipedalism. Specifically, the incremental change of bipedalism could have aided the actual survival of the animal unit through its adaption to new environments, avoidance of predators, conservation/gaining of more nutriments, and successful protection of the progeny by the parental unit. It is possible that bipedalism provided a variety of benefits to the hominin species (Napier, 1964; Sigmon, 1971).

Furthermore, there are several paths through which evolution could have benefited the survival of the ancestors. However, the hominins are the only bipedal species out of all of the great apes (Harcourt-Smith 2007). Therefore, it is important to note that this change was advantageous for the humans but not advantageous for the other great apes. A retracing of the evolutionary traits backwards in an evolutionary timeline from the modern Homo sapiens to the Homo heidelbergensis, H. erectus, Australopithecus and Ardipithecus revealed that the latter species had a closer resemblance to the last common ancestor of chimpanzees and humans. The unsettled dispute lied with the earliest bipedal hominin, which could be Sahelanthropus, Ardipithecus or Orrorin $(\mathrm{Su}$ 2013). In addition, several studies have indicated that the extinct hominin Ardipithecus, which was extremely similar to the common chimpanzee ancestor, possessed the ability to walk on two feet, while spending time in the trees. Indeed, the first hominin, or the first common ancestor was partially bipedal, i.e., it possessed a limited ability to walk on two feet.

The following paragraphs will review several prominent theories of bipedalism. The different models of bipedalism will be examined in accordance with the factors of natural selection. Then a comprehensive approach based on an evolutionary timeline of other great apes in the Hominini tribe and even in the Homininae subfamily will be explained considering the aforementioned perspective of multiple answers.

\section{The savanna-based theory}

The savanna-based theory was one of the earliest models to explain the origins of bipedalism and gathered support from several anthropologists (Dart 1925). It mainly suggested that the early hominids were forced to adapt to an open savanna after they left the trees by walking erect on two feet (Shreeve 1996). According to this theory, the evolution of bipedal locomotion would have been helpful in a savanna because the posture would allow hominins to watch over tall grasses, hunt effectively, or be aware of predators. Unfortunately, the paleoclimatological evidence has argued against the savanna-based theory, and the fossil record shows that the early bipedal hominines were still adapted to climbing trees as well. Several researches have indicated that bipedalism evolved in trees.

\section{The postural feeding hypotheses}

The second model is the postural feeding hypothesis, which has been proposed by Kevin Hunt at Indiana University. This stated that bipedal movements might have evolved into regular habits because they were convenient for obtaining food and keeping balance (Hunt 1994). It has been observed that chimps were only bipedal when they ate. Chimpanzees would reach up for fruit hanging from the trees, and orangutans used their hands to stabilize themselves, while navigating thinner branches (Stanford 2006). Australopithecus afarensis had hand and shoulder features that demonstrated hanging habits, whereas their hip and hind limb clearly indicated bipedalism. 
Because a bipedal posture was utilized for grabbing from an overhead branch and harvesting food, Hunt argues that bipedalism evolved more as a feeding posture than as a walking posture.

\section{Review: Introducing a new perspective}

Retrace the steps back to the common ancestor showed clues to address these perplexing issues and theories. The earliest hominins, Sahelanthropus and Ardipithecus have been suggested to have been bipedal and partly arboreal (Nelson 2013). Interestingly, extinct hominins that were close to the common chimpanzee ancestor were partially bipedal. The evolutionary momentum gradually pushed the common ancestor, which was limitedly bipedal and arboreal, to become chimps that were mostly arboreal with limited bipedal motion (quadrupedal mostly on ground) in one branch and hominins that were mostly terrestrial with full bipedal locomotion in the other evolutionary branch. Evolution, therefore, did not have a single direction from the common ancestor toward Homo sapiens. Ancestors of both chimps and humans that apparently possessed ambiguous traits of humans and chimps evolved in two ways: one toward chimpanzees, which included great chimpanzees and bonobos, and the other toward Homo sapiens (Patterson et al. 2006).

Importantly, hominins slowly evolved to walk like modern humans over a continuous scale. Therefore, the important question was not why the earliest hominins were partially bipedal but rather why hominins became more bipedal over time and replaced their less-bipedal ancestors. This specific evolutionary trait of bipedalism was not necessary for the chimps and their extinct ancestors that lived on the trees.

Despite the alleged lack of evidence, the fact remained that full bipedalism had not been documented in other great apes. Other Hominidae/great apes species, such as gorillas, orangutans, and chimps, but not humans spent much time on the trees. Chimps, for example, were agile climbers and nested on the trees to rest around noon and sleep at night. During the day, gorillas climbed the trees, swung from the branches, and chased one another. Most arboreal great apes, such as orangutans, spent nearly all of their time on the trees. Bipedalism (full bipedalism observed in Homo erectus and modern humans) was not a beneficial trait when moving from one tree to another in an arboreal life.
In addition, a change in environment (moving away from trees) cannot be a cause of the partial bipedalism in early hominins, as suggested by fossil evidence. Nevertheless, when hominins started to settle on the ground, the savannah-based theory could provide an explanation for why hominins evolved to walk like modern humans, replacing their less-bipedal ancestors (Dart 1925). Furthermore, the savannah-based theory incorporated several models of bipedalism, such as the sentinel response, threat display, and endurance running, all of which provided general evidence for how bipedalism aided the survival of hominins in the savannah.

Another model was the postural feeding hypothesis, which was supported by the evidence from several studies. However, there were logical problems associated with it. There were possibly two aspects of bipedalism: i) how were the earliest hominins partially bipedal in the first place?, and ii) why did the hominins become increasingly bipedal over time? Hunt's theory could not explain the second aspect of why partially bipedal hominins evolved to walk like modern humans. $\mathrm{He}$ stated the advantage of obtaining food from branches, or balancing in an arboreal task (Hunt 1992). However, hominins evolved to walk like modern humans on the ground, not on the trees. The evolutionary change that was driven by balancing and reaching on the trees should not have affected the hominins that were abandoning the arboreal life. Hominins continuously evolved to possess terrestrial adaptations and eventually lived on the ground. However, Hunt's theory could explain the origin of bipedalism, i.e., how the last common ancestor of chimpanzees and humans, or why the ancient Miocene hominins were partially bipedal. This theory could explain that some Hominidae became partially bipedal for the benefit of balancing, or obtaining food from branches.

\section{The threat model}

The original proponents of this model theorized that bipedalism originated as a natural defense strategy for early hominids. Hominids were attempting to stay as visible as possible according to instincts of aposematism, or warning displays and intimidation of potential predators (Jablonski and Chaplin 1993). Several morphological and behavioral developments were undertaken to exaggerate visual signals, such as the upright bipedal posture, longer legs, and synchronous body movements. 


\section{The thermoregulatory model}

Peter Wheeler proposed the thermoregulatory model, a model that stated that bipedalism would increase the amount of body surface area, which helped dissipate heat and reduces heat gain (Wheeler 1984). Hominins gained access to more favorable wind speeds and temperatures by being higher above the ground. In addition to reducing the body surface exposed to heat, greater wind flows resulted in a higher temperature loss, which made the organism more comfortable.

Although bipedalism appeared to be a favorable trait, this locomotion in hominins offered certain drawbacks for the survival. The maximum sprint speed of fully bipedal humans was strikingly slower than that of many animals (Powell 2007). Bipedalism was seemingly advantageous, but the surprisingly slow speed observed was attributed to the human anatomy that allowed bipedalism. The average human speed was slower than that of other apes. Such slow speed was a dangerous trait for survival because hominins became vulnerable to carnivores. Full bipedalism was eventually advantageous, but at the beginning, bipedalism exposed humans to the risk of predation. An effective bipedalism theory should state the advantages and explain how a trait that offered an advantage outran potential disadvantages associated with survival.

Other theories that are not mentioned here, in addition to warning display and heat loss, provide a broad reasoning that can be applied not only for hominins and primates but for any animal species. The theory should state not only possible advantages of bipedalism but also why a particular trait would have been selected in hominins over millions of years.

In addition to the primates, several species, such as frogs, snakes, the northern white-faced owl, Phasmatodea, and praying mantis, perform warning displays (Eisner and Grant 1981). However, this does not explain why only humans would walk on two feet to adapt to a warning display. Similar issues remain to be resolved to explain the thermoregulatory model. Thermoregulation (storing fat, panting, estivation, hibernation, etc.) is an important aspect of survival in many species, but not all the animals have become bipedal to lose heat, or to control their body temperature. The two hypotheses did not provide a clear reasoning for why humans were the only species to have achieved bipedalism.

The foregoing theories and other unmentioned theories, such as heat loss, warning, sentinel behavior or running endurance, automatically presume that humans were already adapted to a terrestrial (savannah) life, whereas the earliest hominins were largely tree-dwellers. Several of these theories, therefore, would be incorporated into the savannah-based theory. A few studies have demonstrated that an intimidating visual display and heat loss were possible advantages of bipedalism, but the logic would not remain in an opposite direction.

\section{Overcoming the disadvantage}

The clear advantage of bipedalism was the possibility for ancient hominin species to use their hands. With the evolution of bipedalism, this special advantage was evolved only by the primates. This advantage offered a benefit that overcame the fatal disadvantage of slow speed. Several species could not have utilized their hands for effective provisioning or tool use, even if they had become bipedal through evolution. However, the prehensile hands and feet of primates evolved from the mobile hands of the semi-arboreal tree shrews that lived approximately 100 million years ago and enabled provisioning in ape-like ancestors (Schmidt and Lanz 2004). Similar to humans, modern-day chimpanzees have a limited ability to use their limbs and even sticks to obtain termites in a manner similar to human fishing.

\section{Provisioning model}

The last of prominent bipedalism theories, the provisioning model, was proposed by Owen Lovejoy, who suggested a modified version of Darwin's explanation. What would have been so advantageous about using two hands? Lovejoy proposed that walking on two legs was a main adaptation for pair-bonding to succeed because carrying with two hands was effective for food transport (Lovejoy 1988). Lovejoy's theory also proposed that sexual dimorphism suggested that food gathering would improve the infant survival rate. Males were responsible for provisioning the females, whereas females protected their offspring (Lovejoy 1980). Females would mate exclusively with the provisioning male, and other males would no longer need to fight with each other over the females. Therefore, the males' jagged, blade-like canine teeth diminished over time. Several studies have demonstrated that chimpanzees could carry twice as many nuts in a bipedal position than when walking on all fours (Carvalho et al. 2012). 
Anthropological evidence also supported this theory. The downsizing of male canine teeth, decrease in antagonistic behavior and body size dimorphism corroborated Lovejoy's theory.

\section{CONCLUSION}

As mentioned previously, there could be multiple answers to bipedalism, and there were two aspects of bipedal evolution: (1) that ancient hominins were already partially bipedal, and (2) that hominins evolved full bipedalism. Although the postural feeding theory provided an explanation for the first aspect, the savanna-based theory could provide an answer to why hominins became increasingly bipedal over time. Lovejoy's provisioning model lied between these two theories. Hunt's theory, which suggested that bipedalism involved reaching for food and balancing on the branches, would logically fall before using the hands for provision. The early hominins spent time on the trees, but the species eventually evolved to walk like modern humans on the ground. The evolutionary momentum that was driven by balancing and reaching on the trees should have affected the early hominins. The provisioning model demonstrated how hominins became more bipedal over the time not only by food gathering but also by provisioning infants (monogamy). Nonetheless, the provisioning model did not have sufficient evidence explaining why hominins would have begun to walk like modern humans and lost all adaptations to arboreal life. To this gap was where the savannah-based theory contributed its explanations. When largely bipedal hominins started to settle on the ground, the savannah based-theory would be the explanation for their full bipedalism. The savannah basedtheory included various other models that already assumed that hominins started to live a terrestrial life, such as sentinel behavior, threat, running endurance and thermoregulatory models. The general order of the theories was the following: postural-feeding, provisioning, and savannahbased theories. However, there were no straight lines between these theories, and it was possible that the three forces worked together at one point.

\section{Biological Analogy of Bipedal evolution}

First, there were arboreal hominids that possessed ambiguous traits of bipedalism. These were gradually replaced by two lines of species: one consisting of Pan species and the other comprising hominins. Hominin-like species and modern chimp-like species gradually evolved to undergo specific adaptations to live on the ground and on the trees, and the new hominins presented a survival advantage over their common ancestors. The ambiguous traits were eliminated through the choice and selection. By narrowing its anatomical/biological focus over the evolutionary time, one branch became more bipedal, while the other adapted to a quadrupedal locomotion. However, when the split between the two species became clear, the hominins and chimpanzees would not have competed for resources.

The stated biological relationship between the chimps and humans was similar to the remarkable relationship between the okapi and the giraffe. Similar to the unique adaptation of bipedal locomotion that was only observed in Homo species, the giraffe's long neck was also an evolutionary product exclusive to this species (Badlangana et al. 2007). The okapi and the giraffe are currently the only living members of the Giraffidae family. Although the short-necked okapi's outer appearance resembles a zebra, the okapi is the closest surviving species to the giraffe. Apparently, Darwinian natural selection has led the ancestral giraffes with long necks to reproduce and pass on their genes because they had a competitive advantage that enabled them to reach higher branches. Consequently, the giraffe ancestors fed on acacia leaves and spread through the savannah where tall trees grew. In contrast, the long-neck adaptations became futile for the okapi species, which, ultimately, inhabited canopy forests and fed on the buds, grasses, ferns, fruits, or fungi (Hart and Hart 1989).

\section{REFERENCES}

Auletta G, Leclerc M, Martínez Rafael A. Biological evolution: facts and theories: a critical appraisal 150 years after "The origin of species". Roma: Gregorian \& Biblical Press; 2011.

Ayala FJ. Darwin's greatest discovery: Design without designer. Proc Acad Sci. U.S.A. 2007: 104(Suppl 1):8567-8573.

Badlangana N, Bhagwandin A, Fuxe K, Manger P. Observations on the giraffe central nervous system related to the corticospinal tract, motor cortex and spinal cord: What difference does a long neck make? Neuroscience. 2007;148(2):522-534. 
Dart RA. Australopithecus africanus: The Man-Ape of South Africa. Nature. 1925; 115(2884):195-199.

Darwin C. On the origin of species by means of natural selection: The preservation of favored races in the struggle for life. New York: Heritage Press; 1963.

Eisner T, Grant R. Toxicity, odor aversion, and "olfactory aposematism". Science. 1981; 213(4506):476

Harcourt-Smith WEH. The Origins of Bipedal Locomotion. Handbook of Paleoanthropology. 2013:1-36.

Hart JA, Hart TB. Ranging and feeding behaviour of okapi (Okapia johnstoni) in the Ituri Forest of Zaire: food limitation in a rain-forest herbivore. Symposium of the Zoological Society of London. 1989;61:31-50.

Hunt KD. Positional behavior of Pan troglodytes in the Mahale Mountains and Gombe Stream National Parks, Tanzania. Am J Phys Anthropol. 1992;87(1):83-105.

Hunt KD. The evolution of human bipedality: ecology and functional morphology. J Hum Evol. 1994;26(3):183-202.

Jablonski NG, Chaplin G. Origin of habitual terrestrial bipedalism in the ancestor of the Hominidae. J Hum Evol. 1993;24(4):259-280.

Khanna DR. Human evolution. New Delhi: Discovery Publishing House; 2004.

Lovejoy CO. Hominid Origins-The Role of Bipedalism. Am J Phys Anthropol. 1980;52(2):250-250

Lovejoy CO. Evolution of Human Walking. Sci Am. 1988; 259(5):118-125.

Miller GT. Environmental science: working with the Earth. Belmont, CA: Wadsworth Pub.; 1995.

Napier JR. (1964). The evolution of bipedal walking in the hominids. Liege.

Nelson SV. Chimpanzee fauna isotopes provide new interpretations of fossil ape and hominin ecologies. $P$ Roy Soc Lond B Bio. 2013; 280(1773):20132324.

Patterson N, Richter DJ, Gnerre S, Lander ES, Reich D. Genetic evidence for complex speciation of humans and chimpanzees. Nature. 2006;441(7097):11031108.
Powell A. Humans hot, sweaty, natural-born runners. [2007; cited 2014 Nov 12]. Harvard Gazette. Available from: http://news.harvard.edu/gazette/story/2007/04/human s-hot-sweaty-natural-born-runners/

Schmidt H and Lanz, U. (2004). Surgical anatomy of the hand. Stuttgart: Thieme.

Shreeve, J. Sunset on the Savanna. [1996; cited 2014 Aug 11]. Discover Magazine. Available from: http://discovermagazine.com/1996/jul/sunsetonthesav an818

Sigmon BA. Bipedal behavior and the emergence of erect posture in man. Am J Phys Anthropol. 1971; 34(1), 55-60.

Stanford CB. Arboreal bipedalism in wild chimpanzees: Implications for the evolution of hominid posture and locomotion. Am J Phys Anthropol. 2006;129(2):225231.

Su DF. The Earliest Hominins: Sahelanthropus, Orrorin, and Ardipithecus. [2013; cited 2014 Aug11]. Nature Publishing Group. Available from from: http://www.nature.com/scitable/knowledge/ library/the-earliest-hominins-sahelanthropus-orrorinand-ardipithecus-67648286

Thorpe SKS, Holder RL, Crompton RH. Origin of Human Bipedalism as an Adaptation for Locomotion on Flexible Branches. Science. 2007Jan;316(5829):1328-1331.

Tuttle RH. Human evolution. [2015; cited 2015 Aug.11]. Encyclopedia Britannica. Available from from: http://www.britannica.com/EBchecked/ topic/275670/human-evolution/250597/Theories-ofbipedalism

Wheeler P. The evolution of bipedality and loss of functional body hair in hominids. J Hum Evol. 1984;13(1):91-98.

Wright, S. The roles of mutation, inbreeding, crossbreeding, and selection in evolution. Proceedings of the VI International Congress of Genetrics; 1932. v. 1. p. 356-366. 